\title{
The Correlation of Routine Hematological Parameters with In-hospital Mortality and Length of Hospital Stay in Patients with Large Middle Cerebral Artery Infarction
}

\author{
Muzaffer Güneş ${ }^{1}$ \\ 1. Neurology, Aksaray University Training and Research Hospital, Aksaray, TUR
}

Corresponding author: Muzaffer Güneş, drmuzaffergunes@gmail.com

\section{Abstract \\ Objective}

Neutrophil-to-lymphocyte ratio (NLR) and red blood cell distribution width (RDW) reflect inflammation and these parameters have not been studied in patients with large cerebral artery infarction. This study investigated the correlation of these inflammation parameters with in-hospital mortality and length of hospital stay (LOS) in patients with large middle cerebral artery (MCA) infarction.

\section{Materials and methods}

The study was conducted with patients who had large MCA (M1 and M2 segments) infarction. Clinical data of the patients and laboratory results at presentation were obtained from our database and recorded for statistical analysis. Multivariate logistic regression analysis was used to investigate the prognostic factors. The correlation between hematological parameters and LOS was investigated using Spearman's Rho and Pearson correlation tests.

\section{Results}

A total of 143 patients (48 patients with hospital mortality, 95 patients without hospital mortality) were included in the study. The median LOS in patients with hospital mortality [10 (2-90) days] was significantly higher compared to the patients without [7 (2-30) days] $(\mathrm{p}=0.024)$. RDW-SD was found to be a poor prognostic factor according to the multivariate logistic regression model $(\mathrm{p}=0.004)$. There was a significant but weak and positive correlation between LOS and NLR $(p=0.003, r: 0.248)$ and a significant but weak and negative correlation between LOS and eosinophil count $(\mathrm{p}=0.001, \mathrm{r}:-0.278)$.

\section{Conclusions}

Received 04/15/2020 Review began 04/16/2020 Review ended 04/20/2020 Published 04/29/2020

\section{๑) Copyright 2020}

Güneș. This is an open access article distributed under the terms of the Creative Commons Attribution License CC-BY 4.0., which permits unrestricted use, distribution, and reproduction in any medium, provided the original author and source are credited.
High RDW at presentation is a poor prognostic factor in terms of in-hospital mortality in patients with large MCA infarction. In addition, a positive correlation has been found between NLR and LOS.

\author{
Categories: Neurology \\ Keywords: acute ischemic stroke, length of hospital stay, middle cerebral artery infarction, in-hospital mortality,
} hematological parameters

\section{Introduction}

There are many factors that affect in-hospital mortality in patients with acute ischemic stroke (AIS) [1]. These include the patient's age and severity of AIS, which are the strongest determinants of prognosis in the acute phase of stroke [2]. It is also known that routine hematological parameters affect the prognosis of AIS [3]. Prolonged length of hospital stay (LOS) can result in additional complications in these patients. Previous studies have shown that LOS is associated with many factors such as hypertension, atrial fibrillation, smoking, ischemic heart disease, diabetes mellitus, severity of stroke, inpatient complications, subtype of stroke and age [4-6]. Studies have reported a relationship between neutrophil-to-lymphocyte ratio (NLR) and LOS in some diseases and medical condition, such as acute appendicitis, colorectal cancer surgery $[7,8]$. However, the correlation of routine hematological parameters with in-hospital mortality and LOS was not investigated in patients with large middle cerebral artery (MCA) infarction.

In patients with AIS, the prognosis can get worse as a result of complications that may develop due to prolonged LOS, which may also cause additional financial burden. There is no doubt that knowledge of the factors that affect mortality and LOS is critical. Therefore, this study was conducted to investigate the correlation of routine hematological parameters with in-hospital mortality and LOS in patients with large MCA infarction. 


\section{Materials And Methods \\ Subjects and study design}

This study was conducted retrospectively with patients who were under follow-up and treatment for AIS in the Neurology Department of Aksaray University Training and Research Hospital between August 2014 and January 2018. Patients aged 18 years and older who were admitted to the hospital within the first 24 hours after the onset of symptoms, had M1 and M2-MCA infarction, and did not have any history of mechanical thrombectomy or intravenous thrombolytic therapy were included in the study. Patients with cerebral infarcts other than large MCA infarct (M1 and M2 occlusion), those who were not admitted to the hospital within the first 24 hours after the onset of symptoms, those under 18 years of age, pregnant women, patients who had hemorrhagic infarction, blood disorders, kidney and liver failure, malignant disease, those who received permanent immunomodulatory therapy, and those who had missing data were excluded from the study.

The patients underwent parenchymal and vascular imaging of the brain at presentation. The veins with occlusion were identified according to the cerebrovascular anatomical classification [9]. The subjects were divided into two groups, i.e. those who died of large MCA infarction in the first group and those who did not die of the same cerebrovascular occlusion in the second group (control group).

When patients are brought to the emergency department of our hospital with the preliminary diagnosis of stroke, the first step is to promptly take medical history from the patients or their relatives. The vital signs (arterial oxygen saturation, temperature, heart rate, blood pressure) are also measured rapidly and blood glucose level is measured from the fingertip. The specimens for hematology tests and biochemical analyses are obtained while conducting a swift neurological examination. Computed tomography (CT) or magnetic resonance (MR) imaging of the brain is used to obtain parenchymal images of the brain. In addition, CT angiography or MR angiography of the brain is performed for vascular imaging of the brain. The vein with occlusion is determined by parenchymal and vascular imaging of the brain and subsequently treated.

The laboratory data at the time of presentation to the emergency department was used in this study. Peripheral venous blood samples were obtained from all patients for a complete blood count and blood cell count analyses were conducted using an autoanalyzer (Sysmex XN-1000 hematology analyzer, Kobe, Japan) after centrifugation. NLR was calculated by dividing the neutrophil count by the lymphocyte count, and monocyte-to-lymphocyte ratio (MLR) by dividing the monocyte count by the lymphocyte count.

Clinical and laboratory findings at the time of presentation to the emergency department, results of the parenchymal and vascular brain imaging tests, risk factors and other demographic characteristics were obtained from our database and recorded for statistical analysis. The study was approved by the local authorities and conducted in compliance with the Declaration of Helsinki.

\section{Statistical analysis}

Results are presented as mean \pm standard deviation for normally distributed data, median (min-max) for abnormally distributed data and percentage (\%). To investigate the distribution pattern of the data, Kolmogorov-Smirnov normality test was used. The normally distributed variables were compared using Student's independent samples T test, and abnormally distributed variables were compared using MannWhitney U test. To investigate the prognostic factors of large MCA infarction, univariate and multivariate logistic regression analysis were used. The variables with a P-value of primary comparison less than 0.25 were included in the univariate logistic regression model. Moreover, the variables with a P-value of univariate logistic regression analysis less than 0.1 were included in the multivariate logistic regression model. Hosmer-Lemeshow goodness of fit statistics were used to evaluate the model fit. Cox and Snell pseudo-R2 and Nagelkerke pseudo-R2 tests were used to assess the consistency between the variables. The correlation between two continuous variables was investigated using Spearman's Rho correlation test and Pearson correlation test for variables without and with a normal distribution, respectively. The results were interpreted in accordance with the published Cohen's principles (weak positive correlation, $\mathrm{r}=0.10-0.29$; moderate positive correlation, $r=0.30-0.49$; strong positive correlation, $r=0.50-1.00$ ) [10]. For statistical analysis of all data, SPSS 23.0 software was used for Windows (IBM Corp., Armonk, NY). A P-value less than 0.05 was considered statistically significant.

\section{Results}

One hundred and forty-three patients with large MCA infarction were included in the study. The patients with hospital mortality consisted of 48 patients ( 19 males and 29 females, mean age: $76.3 \pm 10.81$ years) and the patients without hospital mortality consisted of 95 patients ( 47 males and 48 females, median age: 70.4 \pm 11.02 years). The mortality rate of large MCA infarction was $33.6 \%$ (48 out of 143 patients). The gender distribution of the patients with and without hospital mortality was not significantly different $(p=0.263, X 2$ $=1.25$ ), however, the mean age of the patients with hospital mortality was significantly greater compared to the patients without $(\mathrm{p}=0.003)$. 


\section{Cureus}

The comparison of various blood parameters between the groups was presented in Table 1. In the Student's T test, the mean corpuscular volume (MCV), urea and potassium did not significantly differ between the patients with and without hospital mortality $(\mathrm{p}=0.525, \mathrm{p}=0.345$ and $\mathrm{p}=0.694$, respectively). However, the mean red blood cell distribution width (RDW)-SD was significantly higher in patients with hospital mortality, compared to the patients without $(\mathrm{p}=0.004)$. According to Mann-Whitney U test, the median Creactive protein (CRP), white blood cell (WBC), neutrophil, monocyte, red blood cell (RBC), hemoglobin, hematocrit, RDW-CV, platelet, creatinine, sodium, alanine aminotransferase (ALT) and aspartate aminotransferase (AST) values did not significantly differ between the patients with and without hospital mortality $(\mathrm{p}=0.697, \mathrm{p}=0.366, \mathrm{p}=0.069, \mathrm{p}=0.615, \mathrm{p}=0.469, \mathrm{p}=0.229, \mathrm{p}=0.417, \mathrm{p}=0.137, \mathrm{p}=0.434, \mathrm{p}=$ $0.667, p=0.364, p=0.724$ and $p=0.937$, respectively). However, the median NLR and MLR were significantly higher $(\mathrm{p}=0.001)$, and the median lymphocyte and eosinophil values were significantly lower in patients with hospital mortality $(\mathrm{p}<0.001$ and $\mathrm{p}=0.028)$, compared with the patients without.

\begin{tabular}{|c|c|c|c|}
\hline & Patients without hospital mortality & Patients with hospital mortality & P-value \\
\hline Age & $70.44 \pm 11.02$ & $76.29 \pm 10.81$ & 0.003 \\
\hline RDW-SD, fL & $43.64 \pm 5.55$ & $46.75 \pm 6.85$ & 0.004 \\
\hline Mean corpuscular volume, $\mathrm{fL}$ & $87.17 \pm 6.9$ & $87.96 \pm 7.24$ & 0.525 \\
\hline Urea, mg/dL & $42.28 \pm 16.55$ & $45.06 \pm 17.11$ & 0.345 \\
\hline Potassium, mmol/L & $4.27 \pm 0.48$ & $4.3 \pm 0.49$ & 0.694 \\
\hline C-reactive protein, mg/dL & $5.85(0.22-79.66)$ & $6.21(0.54-154)$ & 0.697 \\
\hline White blood cell, $10^{9} / \mathrm{L}$ & $9.49(5.25-19.8)$ & $9.62(4.7-22.93)$ & 0.366 \\
\hline Neutrophil, $10^{9} / \mathrm{L}$ & $6.23(2.53-14.4)$ & $6.82(2.33-20.77)$ & 0.069 \\
\hline Lymphocyte, $10^{9} / \mathrm{L}$ & $2.22(0.59-6.92)$ & $1.57(0.52-4.58)$ & $<0.001$ \\
\hline NLR & 2.68 (0.92-21.42) & $4.43(1.18-24.36)$ & 0.001 \\
\hline Monocyte, $10^{9} / \mathrm{L}$ & $0.58(0.12-1.49)$ & $0.61(0.2-1.32)$ & 0.615 \\
\hline MLR & $0.27(0.4-0.88)$ & $0.36(0.11-1.48)$ & 0.001 \\
\hline Eosinophil, $10^{9} / \mathrm{L}$ & $0.9(0.002-2.37)$ & $0.055(0.001-0.45)$ & 0.028 \\
\hline Red blood cell, $10^{12} / \mathrm{L}$ & $4.8(3.17-7.66)$ & $4.74(3.3-6.03)$ & 0.469 \\
\hline Hemoglobin, g/dL & $13.7(6.9-18.5)$ & $13.65(10.1-16.9)$ & 0.229 \\
\hline Hematocrit, \% & $41.9(24.2-58.2)$ & $41.25(29.3-50)$ & 0.417 \\
\hline RDW-CV, \% & $13.6(10.3-26)$ & $14.35(10-23.6)$ & 0.137 \\
\hline Platelet, $10^{9} / \mathrm{L}$ & $232(135-515)$ & 225 (102-399) & 0.434 \\
\hline Creatinine, mg/dL & $0.91(0.42-3.4)$ & $0.9(0.46-1.34)$ & 0.667 \\
\hline Sodium, mmol/L & $140(131-146)$ & 139 (134-145) & 0.364 \\
\hline ALT, U/L & $14(5-65)$ & $15(4-39)$ & 0.724 \\
\hline AST, U/L & $20(9-52)$ & $20(12-43)$ & 0.937 \\
\hline
\end{tabular}

\section{TABLE 1: The comparison of blood parameters between the groups.}

RDW: Red blood cell distribution width; NLR: Neutrophil / lymphocyte ratio; MLR: Monocyte / lymphocyte ratio; ALT: Alanine aminotransferase; AST: Aspartate aminotransferase; SD: Standard deviation; CV: Coefficient of variation.

The Chi-square test revealed that the rates of diabetes mellitus, arterial hypertension, hyperlipidemia, congestive heart failure, coronary artery disease, valve disease and atrial fibrillation did not significantly differ between the patients with and without hospital mortality $(p=0.402, p=0.746, p=0.228, p=0.058, p=$ $0.497, \mathrm{p}=0.476$ and $\mathrm{p}=0.557$, respectively) (Table 2 ). 


\section{Cureus}

\begin{tabular}{|c|c|c|c|c|}
\hline & Patients without hospital mortality $(n=95)$ & Patients with hospital mortality $(n=48)$ & P-value & $\mathrm{X}^{2}$ value \\
\hline Gender (M/F) & $47 / 48$ & $19 / 29$ & 0.263 & 1.25 \\
\hline Diabetes Mellitus & $29(30.5 \%)$ & $18(37.5 \%)$ & 0.402 & 0.7 \\
\hline Hypertension & $75(78.9 \%)$ & $39(81.3 \%)$ & 0.746 & 0.1 \\
\hline Hyperlipidemia & $22(23.2 \%)$ & $7(14.6 \%)$ & 0.228 & 1.45 \\
\hline Congestive heart failure & $17(17.9 \%)$ & $3(6.25 \%)$ & 0.058 & 3.59 \\
\hline Coronary artery disease & $16(16.8 \%)$ & $6(12.5 \%)$ & 0.497 & 0.46 \\
\hline Valve disease & $1(1.05 \%)$ & $0(0 \%)$ & 0.476 & 0.51 \\
\hline Atrial fibrillation & $24(25.3 \%)$ & $10(20.8 \%)$ & 0.557 & 0.34 \\
\hline
\end{tabular}

TABLE 2: The comparison of categorical variables (presence) between the groups.

Table 3 represents the univariate and multivariate logistic regression analysis results. In the univariate logistic regression model, the age, RDW-SD, neutrophil, lymphocyte, NLR and MLR were prognostic factors $(\mathrm{p}=0.004, \mathrm{p}=0.006, \mathrm{p}=0.012, \mathrm{p}=0.002, \mathrm{p}=0.001$ and $\mathrm{p}<0.001$, respectively). However, only RDW-SD and the presence of congestive heart failure were found to be prognostic factors in the multivariate logistic regression model $(\mathrm{p}=0.004$ and $\mathrm{p}=0.03$, respectively).

\begin{tabular}{|c|c|c|c|c|}
\hline & \multicolumn{2}{|l|}{ Univariate } & \multicolumn{2}{|l|}{ Multivariate } \\
\hline & OR $(95 \% \mathrm{Cl})$ & P-value & OR $(95 \% \mathrm{Cl})$ & P-value \\
\hline Age & $1.053(1.016-1.091)$ & 0.004 & $1.033(0.993-1.075)$ & 0.11 \\
\hline RDW-SD & $1.09(1.025-1.161)$ & 0.006 & $1.112(1.035-1.196)$ & 0.004 \\
\hline Neutrophil & $1.155(1.032-1.293)$ & 0.012 & $1.073(0.848-1.358)$ & 0.558 \\
\hline Lymphocyte & $0.513(0.338-0.778)$ & 0.002 & $0.786(0.408-1.516)$ & 0.473 \\
\hline NLR & $1.199(1.079-1.332)$ & 0.001 & $1.065(0.835-1.357)$ & 0.614 \\
\hline MLR & $24.34(4.21-140.43)$ & $<0.001$ & $3.734(0.372-37.436)$ & 0.263 \\
\hline Congestive heart failure & $3.269(0.908-11.771)$ & 0.07 & $5.769(1.183-28.139)$ & 0.03 \\
\hline Eosinophil & $0.106(0.005-2.05)$ & 0.137 & - & - \\
\hline Hemoglobin & $0.912(0.764-1.088)$ & 0.307 & - & - \\
\hline RDW-CV & $1.052(0.906-1.221)$ & 0.504 & - & - \\
\hline \multirow[t]{3}{*}{ Hyperlipidemia } & $1.765(0.695-4.485)$ & 0.232 & - & - \\
\hline & & & \multicolumn{2}{|c|}{ Cox and Snell pseudo- $R^{2}=0.236$} \\
\hline & & & \multicolumn{2}{|c|}{ Nagelkerke pseudo- $R^{2}=0.328$ Hosmer-Lemeshow $P=0.1$} \\
\hline
\end{tabular}

TABLE 3: The univariate and multivariate logistic regression analysis results.

RDW: Red blood cell distribution width; NLR: Neutrophil / lymphocyte ratio; MLR: Monocyte / lymphocyte ratio; OR: Odds ratio.

The overall mean LOS was $11.6 \pm 12.74$ days (range: $2-90$ days). The median LOS in patients with hospital mortality [10 (2-90) days] was significantly higher compared to the patients without [7 (2-30) days] ( $\mathrm{p}=$ 0.024). Table 4 presents the results of the correlations between the blood test parameters and the LOS. According to the Spearman test, a significant but weak, positive correlation of the LOS with neutrophil $(\mathrm{p}=$ 0.039, rho: 0.173) and NLR ( $p=0.003$, rho: 0.248$)$ was evident. In addition, a significant but weak negative correlation of the LOS with lymphocyte ( $\mathrm{p}=0.002$, rho: -0.253$)$, eosinophil $(\mathrm{p}=0.001$, rho: -0.278 ) and ALT 


\section{Cureus}

$(p=0.037$, rho: -0.175$)$ was evident (Figure 1).

\begin{tabular}{|c|c|c|}
\hline & \multicolumn{2}{|l|}{ Length of hospital stay } \\
\hline & Correlation coefficient $(r)$ & P-value \\
\hline Age & 0.164 & 0.05 \\
\hline Red blood cell distribution width-SD & 0.12 & 0.152 \\
\hline Mean corpuscular volume & 0.044 & 0.598 \\
\hline C-reactive protein & 0.112 & 0.185 \\
\hline White blood cell & 0.108 & 0.201 \\
\hline Neutrophil & 0.173 & 0.039 \\
\hline Lymphocyte & -0.253 & 0.002 \\
\hline Neutrophil / lymphocyte ratio & 0.248 & 0.003 \\
\hline Monocyte & -0.065 & 0.442 \\
\hline Monocyte / lymphocyte ratio & 0.134 & 0.11 \\
\hline Eosinophil & -0.278 & 0.001 \\
\hline Red blood cell & -0.03 & 0.722 \\
\hline Hemoglobin & -0.23 & 0.787 \\
\hline Hematocrit & -0.039 & 0.647 \\
\hline Red blood cell distribution width -CV & 0.059 & 0.486 \\
\hline Platelet & -0.085 & 0.312 \\
\hline Creatinine & 0.095 & 0.260 \\
\hline Sodium & -0.012 & 0.888 \\
\hline Alanine aminotransferase & -0.175 & 0.037 \\
\hline Aspartate aminotransferase & 0.013 & 0.881 \\
\hline Urea & 0.03 & 0.718 \\
\hline Potassium & 0.017 & 0.836 \\
\hline
\end{tabular}

TABLE 4: Correlations between the blood test parameters and length of hospital stay.

SD: Standard deviation. 


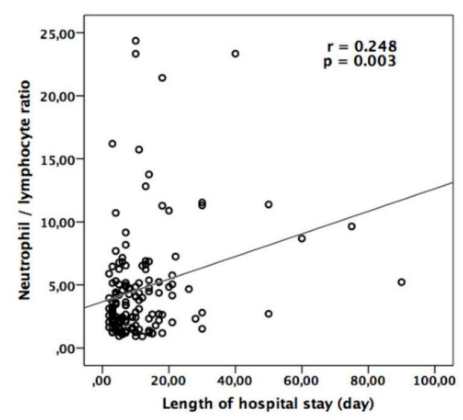

$\mathrm{C}$

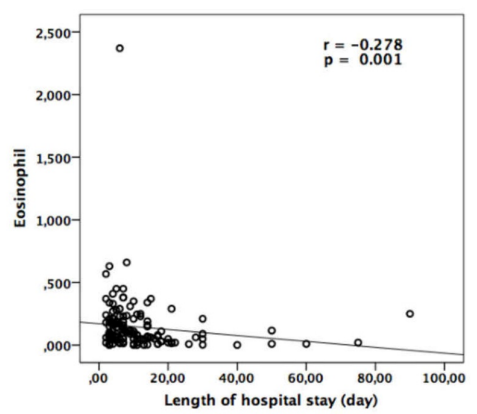

B

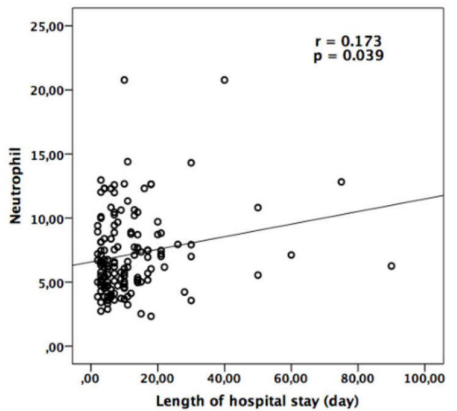

D

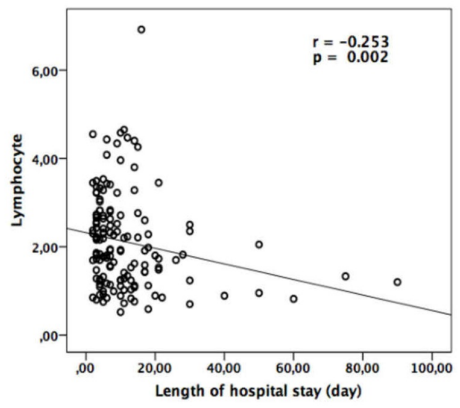

FIGURE 1: Relationship between length of hospital stay and neutrophil / lymphocyte ratio (NLR), neutrophil, eosinophil and lymphocyte.

\section{Discussion}

This study has shown that high RDW is a poor prognostic factor for in-hospital mortality in patients with large MCA infarction. Moreover, it was also shown that there was a significant and positive correlation between NLR and neutrophil count at presentation and LOS, and a significant and negative correlation between eosinophil and lymphocyte counts at presentation and LOS. The study was conducted using laboratory data collected in the acute phase of the disease. All subjects were selected from patients with large MCA (M1 and M2 segments) infarction in order to obtain a relatively homogenous population.

RDW is a hematological parameter that reflects the degree of anisocytosis (variation in erythrocyte diameters) [11]. Previous studies suggested that RDW was associated with inflammatory markers such as CRP and sedimentation rate, and therefore it could be used as a parameter that indicates inflammation [12, 13]. Several recent studies reported that high RDW was associated with stroke development and with a poor prognosis in patients who had ischemic stroke $[14,15]$. On the other hand, it was found in another study that RDW did not predict the severity of stroke and functional outcome in the early phase of AIS [16]. In this study, which contained a relatively more homogenous patient population than previous studies, it was found that high RDW at the time of presentation was a poor prognostic marker for in-hospital mortality in patients with large MCA infarction. It was thought that high RDW at the time of presentation could be a poor prognostic marker in patients with large MCA infarction. However, further studies are necessary.

It was reported that LOS was affected by various patient-related factors such as diabetes mellitus, age, atrial fibrillation, severity of stroke, ischemic heart disease and subtype of stroke [4, 5]. Studies investigating the correlation of LOS with hematological parameters and inflammation markers are very scarce. NLR is known as a parameter that indicates subclinical systemic inflammation [17]. According to a study published in the literature, there was a significant positive correlation between NLR and LOS [18]. Similarly, this study also demonstrated a positive correlation between neutrophil count, NLR and LOS, and a negative correlation between lymphocyte count and LOS. This study also showed a negative correlation between LOS and eosinophil count. In the light of this information, it was concluded that subclinical systemic inflammation could be a factor that prolongs LOS. 
In the recent years, many studies have shown that a high NLR had a negative impact on the prognosis of AIS [19-21]. However, these studies encompassed all patients with AIS including lacunar infarction cases that can have a good prognosis and large cerebral infarction cases that can have a poor prognosis. Therefore, these studies did not have a homogenous patient population. On the other hand, this study only included patients with large MCA infarction and had a more homogenous patient population. To the best of our knowledge, there are no studies investigating NLR in patients with large MCA infarction in the literature. Unlike other studies in the literature, this study showed that NLR was a prognostic factor according to the univariate analysis, but not an independent prognostic factor according to the multivariate analysis. This was thought to stem from the homogenous patient population employed in this study. It may also stem from the fact that the study used laboratory data collected in the very early phase of stroke or that there were few patients included in the groups.

The strengths of this study were as follows: it included a relatively more homogenous patient population than the previous studies and used laboratory data that were collected at the time of presentation to the hospital. The study was retrospective, which was a limitation of this study.

\section{Conclusions}

In conclusion, high RDW at the time of admission is useful and independent poor prognostic marker for inhospital mortality of patients with large MCA infarction. There is a relationship between routine hematological parameters and LOS, but this relationship is weak. Even so, it is of course important to know the relationship between LOS and disease prognosis with these routine hematological parameters. In this respect, there is a need for prospective multicenter studies that include larger patient groups.

\section{Additional Information \\ Disclosures}

Human subjects: Consent was obtained by all participants in this study. T. C. AKSARAY Governorship Provincial Health Directorate issued approval 12/02/2020/ 74114513-771-E.214. Aksaray University Faculty of Medicine In the related refer of Faculty Member Muzaffer GÜNEŞ, it is stated that you plan to apply your research on "The correlation of routine hematological parameters with in-hospital mortality and length of hospital stay in patients with large middle cerebral artery infarction" in Aksaray Training and Research Hospital Neurology Clinic. Your research requests have been evaluated by the "Institutional Review Board" and have been properly evaluated by our Directorate. Dr. Koca ÇALIŞKAN Head of Public Hospitals Services. Animal subjects: All authors have confirmed that this study did not involve animal subjects or tissue. Conflicts of interest: In compliance with the ICMJE uniform disclosure form, all authors declare the following: Payment/services info: All authors have declared that no financial support was received from any organization for the submitted work. Financial relationships: All authors have declared that they have no financial relationships at present or within the previous three years with any organizations that might have an interest in the submitted work. Other relationships: All authors have declared that there are no other relationships or activities that could appear to have influenced the submitted work.

\section{Acknowledgements}

The author(s) would like to thank Dr. A. Baygül and Asst. Prof. Dr. P. Güneş for their assistance in statistical analyses.

\section{References}

1. Gattringer T, Posekany A, Niederkorn K, et al.: Predicting early mortality of acute ischemic stroke: scorebased approach. Stroke. 2019, 50:349-356. 10.1161/STROKEAHA.118.022863

2. Weimar C, König IR, Kraywinkel K, Ziegler A, Diener HC: Age and National Institutes of Health Stroke Scale Score within 6 hours after onset are accurate predictors of outcome after cerebral ischemia: development and external validation of prognostic models. Stroke. 2004, 35:158-162. 10.1161/01.STR.0000106761.94985.8B

3. Fan L, Gui L, Chai EQ, Wei CJ: Routine hematological parameters are associated with short- and long-term prognosis of patients with ischemic stroke. J Clin Lab Anal. 2018, 32:e22244. 10.1002/jcla.22244

4. Huang YC, Hu CJ, Lee TH, Yang JT, Weng HH, Lin LC, Lai SL: The impact factors on the cost and length of stay among acute ischemic stroke. J Stroke Cerebrovasc Dis. 2013, 22:152-158. 10.1016/j.jstrokecerebrovasdis.2012.10.014

5. Koton S, Bornstein NM, Tsabari R, Tanne D: Derivation and validation of the prolonged length of stay score in acute stroke patients. Neurology. 2010, 74:1511-1516. 10.1212/WNL.0b013e3181dd4dc5

6. Appelros P: Prediction of length of stay for stroke patients . Acta Neurol Scand. 2007, 116:15-19. 10.1111/j.1600-0404.2006.00756.x

7. Kelly ME, Khan A, Riaz M, et al.: The utility of neutrophil-to-lymphocyte ratio as a severity predictor of acute appendicitis, length of hospital stay and postoperative complication rates. Dig Surg. 2015, 32:459463. 10.1159/000440818

8. Gohil R, Rishi M, Tan BH: Pre-operative serum albumin and neutrophil-lymphocyte ratio are associated with prolonged hospital stay following colorectal cancer surgery. Br J Med Med Res. 2014, 4:481-487.

10.9734/BJMMR/2014/5444\#sthash.FpqnVF8X.dpuf 
9. Chandra A, Li WA, Stone CR, Geng X, Ding Y: The cerebral circulation and cerebrovascular disease I: anatomy. Brain Circ. 2017, 3:45-56. 10.4103/bc.bc_10_17

10. Cohen J: Statistical Power Analysis for the Behavioral Sciences. Routledge, Abington, UK; 1988.

11. Salvagno GL, Sanchis-Gomar F, Picanza A, Lippi G: Red blood cell distribution width: a simple parameter with multiple clinical applications. Crit Rev Clin Lab Sci. 2015, 52:86-105. 10.3109/10408363.2014.992064

12. Hu ZD, Chen Y, Zhang L, et al.: Red blood cell distribution width is a potential index to assess the disease activity of systemic lupus erythematosus. Clin Chim Acta. 2013, 425:202-205. 10.1016/j.cca.2013.08.007

13. Lippi G, Targher G, Montagnana M, Salvagno GL, Zoppini G, Guidi GC: Relation between red blood cell distribution width and inflammatory biomarkers in a large cohort of unselected outpatients. Arch Pathol Lab Med. 2009, 133:628-632. 10.1043/1543-2165-133.4.628

14. Ani C, Ovbiagele B: Elevated red blood cell distribution width predicts mortality in persons with known stroke. J Neurol Sci. 2009, 277:103-108. 10.1016/j.jns.2008.10.024

15. Kim J, Kim YD, Song TJ, et al.: Red blood cell distribution width is associated with poor clinical outcome in acute cerebral infarction. Thromb Haemost. 2012, 108:349-356. 10.1160/TH12-03-0165

16. Ntaios G, Gurer O, Faouzi M, Aubert C, Michel P: Red cell distribution width does not predict stroke severity or functional outcome. Int J Stroke. 2012, 7:2-6. 10.1111/j.1747-4949.2011.00609.x

17. Yang DH, Qian MZ, Wei MM, et al.: The correlation of neutrophil-to-lymphocyte ratio with the presence and activity of myasthenia gravis. Oncotarget. 2017, 8:76099-76107. 10.18632/oncotarget.18546

18. Zhao L, Dai Q, Chen X, et al.: Neutrophil-to-lymphocyte ratio predicts length of stay and acute hospital cost in patients with acute ischemic stroke. J Stroke Cerebrovasc Dis. 2016, 25:739-744. 10.1016/i.jstrokecerebrovasdis.2015.11.012

19. Celikbilek A, Ismailogullari S, Zararsiz G: Neutrophil to lymphocyte ratio predicts poor prognosis in ischemic cerebrovascular disease. J Clin Lab Anal. 2014, 28:27-31. 10.1002/jcla.21639

20. Qun S, Tang Y, Sun J, et al.: Neutrophil-to-lymphocyte ratio predicts 3-month outcome of acute ischemic stroke. Neurotox Res. 2017, 31:444-452. 10.1007/s12640-017-9707-Z

21. Zhang J, Ren Q, Song Y, He M, Zeng Y, Liu Z, Xu J: Prognostic role of neutrophil-lymphocyte ratio in patients with acute ischemic stroke. Medicine. 2017, 96:8624. 10.1097/MD.0000000000008624 